\title{
PIK3R3 regulates PPARo expression to stimulate fatty acid $\beta$-oxidation and decrease hepatosteatosis
}

\author{
Xi Yang, Yinjia Fu, Fuqing Hu, Xuelai Luo, Junbo Hu and Guihua Wang \\ Phosphatidylinositol 3-kinase (PI3K) signaling plays an important role in the regulation of cellular lipid metabolism and \\ non-alcoholic fatty liver disease (NAFLD). However, little is known about the role of the regulatory subunits of PI3K in lipid \\ metabolism and NAFLD. In this study, we characterized the functional role of PIK3R3 in fasting-induced hepatic lipid \\ metabolism. In this study, we showed that the overexpression of PIK3R3 promoted hepatic fatty acid oxidation via \\ PIK3R3-induced expression of PPAR $\alpha$, thus improving the fatty liver phenotype in high-fat diet (HFD)-induced mice. \\ By contrast, hepatic PIK3R3 knockout in normal mice led to increased hepatic TG levels. Our study also showed that \\ PIK3R3-induced expression of PPAR $\alpha$ was dependent on HNF4 $\alpha$. The novel PIK3R3-HNF4 $\alpha$-PPAR $\alpha$ signaling axis plays a \\ significant role in hepatic lipid metabolism. As the activation of PIK3R3 decreased hepatosteatosis, PIK3R3 can be considered \\ a promising novel target for developing NAFLD and metabolic syndrome therapies. \\ Experimental \& Molecular Medicine (2018) 50, e431; doi:10.1038/emm.2017.243; published online 19 January 2018
}

\section{INTRODUCTION}

The liver acts as the 'metabolic integrator' of lipid metabolism by regulating lipogenesis, fatty acid oxidation, and lipoprotein uptake and secretion. ${ }^{1}$ Non-alcoholic fatty liver disease (NAFLD) is currently the most common liver disease worldwide and has been estimated to occur in 30\% of adults in North American and Asian populations. NAFLD is characterized by hepatic steatosis, which can subsequently progress to non-alcoholic steatohepatitis (NASH). Further progression from NASH to cirrhosis may cause liver failure and hepatocellular carcinoma (HCC). ${ }^{2}$ Moreover, fatty liver has also been linked to diabetes and other metabolic diseases. Although NAFLD has been associated with decreased fatty acid $\beta$-oxidation and increased lipogenesis, the molecular mechanisms for fatty liver development are not well understood.

Phosphatidylinositol 3-kinase (PI3K)/AKT signaling is involved in cell survival and proliferation. ${ }^{3}$ Recent reports suggest that PI3K/AKT activates transcriptional regulators of lipid metabolism, such as sterol regulatory element-binding proteins (SREBPs), and plays an important role in the regulation of cellular lipid metabolism. ${ }^{4-7}$ Class IA PI3Ks consist of a regulatory subunit and a catalytic subunit. ${ }^{4}$ It has been reported that the hepatic p110 $\alpha$ catalytic subunit of PI3Kknockout mice causes hypolipidemia. ${ }^{8}$ However, little is known about the role of regulatory subunits of PI3K in lipid metabolism.

PIK3R3, a $62 \mathrm{kDa}$ regulatory subunit of PI3K, can bind to the p110 catalytic subunit through the iSH2 domain. ${ }^{9}$ Previously, we reported that PIK3R3 was important for cell proliferation and tumor growth and was overexpressed in several types of cancer. ${ }^{10,11}$ Moreover, we showed that the inhibition of PIK3R3 blocked cell cycle progression, induced cell differentiation and inhibited tumor angiogenesis. ${ }^{10,12-14}$ However, the role of PI3R3 in physiology and lipid metabolism remains largely unknown.

Peroxisome proliferator-activated receptor alpha (PPAR $\alpha)$ is a ligand-activated nuclear receptor that is highly expressed in tissues with high FAO rates, such as the liver, skeletal muscle, heart and brown adipose tissue. ${ }^{2,15,16}$ PPAR $\alpha$ is also a nuclear hormone receptor that functions as a central regulator of hepatic lipid metabolism. Many PPAR $\alpha$-targeted genes have been demonstrated to play key roles at different points of hepatic lipid metabolism, such as uptake, binding, oxidation of fatty acids, lipid droplet biology and ketogenesis. ${ }^{17-19}$ In addition, PPAR $\alpha$ has emerged as a central transcriptional regulator involved in multiple metabolic processes, such as the metabolism of cholesterol, glucose, bile acids and amino acids in the liver. ${ }^{20,21}$

Department of Gastrointestinal Surgery Center, Tongji Hospital, Tongji Medical College, Huazhong University of Science and Technology, Wuhan, China Correspondence: Professor G Wang, Department of Gastrointestinal Surgery Center, Tongji Hospital, Tongji Medical College, Huazhong University of Science and Technology, 1095\# Jiefang Avenue, Wuhan 430030, China.

E-mail: ghwang@tjh.tjmu.edu.cn

Received 27 July 2016; revised 25 May 2017; accepted 28 June 2017 
In this study, we have described a functional link between PIK3R3 and hepatic lipid metabolism and characterized the functional role of PIK3R3 in vivo under fasting and high-fat diet conditions. We showed that the overexpression of PIK3R3 promoted hepatic fatty acid oxidation via PIK3R3-induced expression of PPAR $\alpha$, thus improving the fatty liver phenotype in HFD-fed mice. By contrast, the knockdown of hepatic PIK3R3 in normal mice led to increased hepatic TG levels. Furthermore, PIK3R3-induced expression of PPAR $\alpha$ was HNF4 $\alpha$-dependent. Collectively, PIK3R3 depends on the PIK3R3-HNF4 $\alpha-$ PPAR $\alpha$ axis to regulate lipid metabolism in the liver.

\section{MATERIALS AND METHODS}

\section{Animals and experimental design}

Eight-week-old male C57BL/6J mice were purchased from the Model Animal Research Center of Nanjing University (Nanjing, China). They were housed and maintained on a $12 \mathrm{~h}$ light-dark cycle and received a regular unrestricted diet. Mice were fed either a normal chow ( $9 \%$ fat) or a high-fat diet (HFD) (45\% fat) ad libitum and had free access to water. This study was conducted following the Animal Study Guidelines of Huazhong University of Science and Technology. Mice were injected with Ad-Pik3r3, Ad-Pparo, Ad-control, si-Pik3r3, si-Ppar $\alpha$ or si-control $\left(1.0 \times 10^{9}\right.$ active viral particles or $40 \mathrm{nmol}$ siRNA in $100 \mu \mathrm{l}$ of PBS) twice a week for 2 weeks. Five days after the last injection, the mice were fasted for $6 \mathrm{~h}$, and their livers were collected for further analyses.

\section{Preparation of expression plasmids, recombinant adenoviruses and RNA interference}

The full-length human or mouse PIK3R3/PPAR $\alpha$ (Pik3r3/Ppar $\alpha$ ) gene was amplified by polymerase chain reaction (PCR) from human liver cDNA or C57BL/6J mouse liver cDNA, respectively. Human PIK3R3 or PPAR $\alpha$ was cloned into the pcDNA3.1 vector, and recombinant adenovirus expressing mouse Pik3r3 or Pparo were generated as previously described. RNA interference (targeting PIK3R3, PPAR $\alpha$, HNF4 $\alpha$, Pik3r3 or Ppar $\alpha$ ) and the siRNA negative control (si-control) were synthesized and purified by RiboBio (RiboBio Corporation, Guangzhou, China) (Supplementary Table 1).

\section{Analytical procedures and chemicals}

Concentrations of ketone bodies in serum or cell culture supernatants were determined using a colorimetric diagnostic kit (Cayman Chemical Company, Ann Arbor, MI, USA). Liver TG levels were measured using a colorimetric diagnostic kit (Jiancheng Bioengineering Institute, Nanjing, China).

\section{Cell culture and transfection}

HepG2 and LO2 cell lines were cultured in DMEM containing 10\% FBS and penicillin/streptomycin. Cells were transfected with expression plasmids at a final concentration of $4 \mu \mathrm{g}$ or $100 \mathrm{~nm}$ siRNA using Lipofectamine 2000 (Invitrogen). Media was changed $6 \mathrm{~h}$ after transfection. The relative expression levels of genes were determined $48 \mathrm{~h}$ after transfection.

\section{Quantitative real-time PCR}

Total RNA was isolated from cells or pulverized liver using TRIzol (Invitrogen). Quantitative real-time reverse transcriptase PCR (qRT-PCR) was performed using the SYBR Green I Q-PCR kit
(Transgen Biotech) on an ABI 7300 system. Human or mouse gene expression data were normalized to Gapdh expression levels. All specific primer pairs for each gene are shown in Supplementary Table 2.

\section{Western blot analysis}

Protein was extracted from frozen organ samples or cultured hepatocytes in cell lysis buffer. Approximately $50 \mu \mathrm{g}$ of protein was separated on $10 \%$ SDS-polyacrylamide gel (SDS-PAGE) gels and transferred to PVDF membranes. Western blot detection was performed using antibodies specific for PIK3R3, GAPDH (Santa Cruz Biotechnology, CA, USA), HNF4 $\alpha$ (Cell Signaling Technology, MA, USA), ACADM, CPT1a (Proteintech Group, Chicago, IL, USA), PPAR $\alpha$ or PPAR $\gamma$ (Boster, Wuhan, China), which were previously tested and studied using both human and mouse samples.

\section{Histology and immunohistochemistry}

For H\&E staining, liver tissues were fixed, embedded and cut into sections as previously described ${ }^{14}$ For Oil Red $\mathrm{O}$ staining, liver tissue was frozen in liquid nitrogen and cut into $10-\mu \mathrm{m}$ sections. Sections were stained and analyzed at $\times 100$ magnification using a microscope. Immunohistochemical examination was performed as previously described. ${ }^{14}$

\section{ChIP assay}

ChIP assay kits purchased from Millipore were used according to the manufacturer's protocol. ChIP assays were carried out using an anti-HNF4 $\alpha$ antibody as previously described (Millipore \#17-371, Burlington, MA, USA). RNA-polymerase 11 , which could bind the promoter region of GAPDH, was used as a positive control. Normal rabbit IgG was used as a negative control. The following primers for the PPAR $\alpha$ promoter were used for the ChIP assays: forward $5^{\prime}$ TTGCGTAGGCACAAAGTCAG- ${ }^{\prime}$ and reverse $5^{\prime}$-CCCAGGATGCAA AATAAGGA-3'; the control primers used for GAPDH were provided in the kit.

\section{Statistical analysis}

All data are expressed as the mean \pm s.e.m. (standard error of the mean). Intergroup and intragroup comparisons were conducted using Student's $t$-test and ANOVA, respectively. $P$-values were calculated using an unpaired Student's $t$-test.

\section{RESULTS}

Hepatic PIK3R3 expression is increased after fasting and is downregulated in a high-fat diet mouse model of fatty liver We utilized a high-fat diet-induced fatty liver mouse model (HFD mice, C57BL/6 background). H\&E staining showed that HFD mice had increased fatty liver, and Oil Red $\mathrm{O}$ staining showed that HFD mice had significantly increased hepatic intracellular triacylglycerol accumulation. We also observed that the expression levels of mitochondrial and peroxisomal fatty acid oxidation-related genes, such as carnitine palmitoyl transferase 1a (CPT1a) and medium-chain acyl-CoA dehydrogenase $(A C A D M)$, were regulated by fasting (Figure 1a). Serum concentrations of TG were significantly lower in normal chow-fed mice than in the HFD-fed mice, while the levels of ketone bodies were lower in the HFD-fed mice than in the normal chow-fed mice $(P<0.01)$ (Figure $1 \mathrm{~b})$. To identify the role of PIK3R3 in lipid metabolism, we examined the PIK3R3 
a
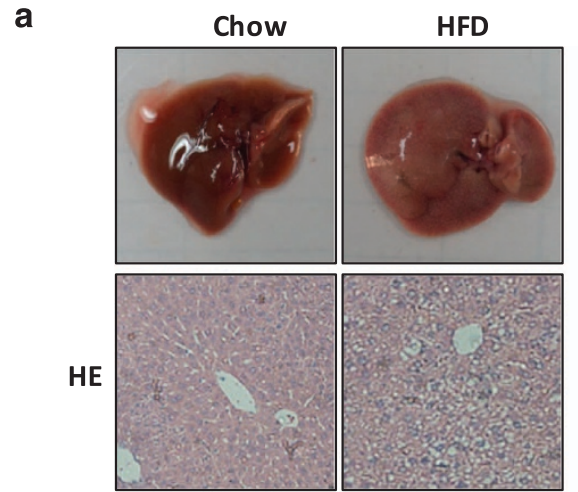

Oil red

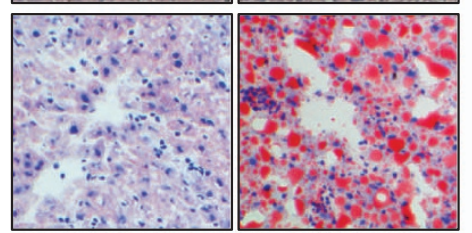

Cpt1a
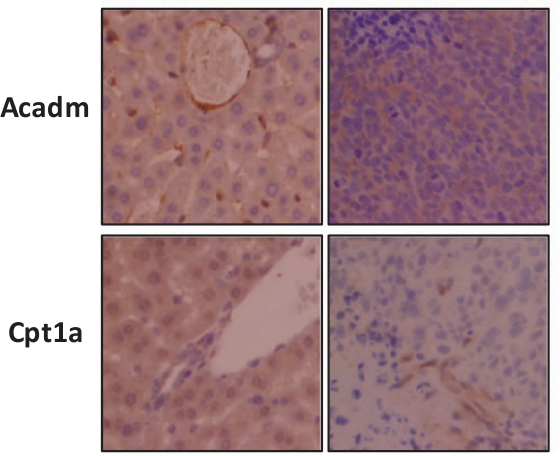

b
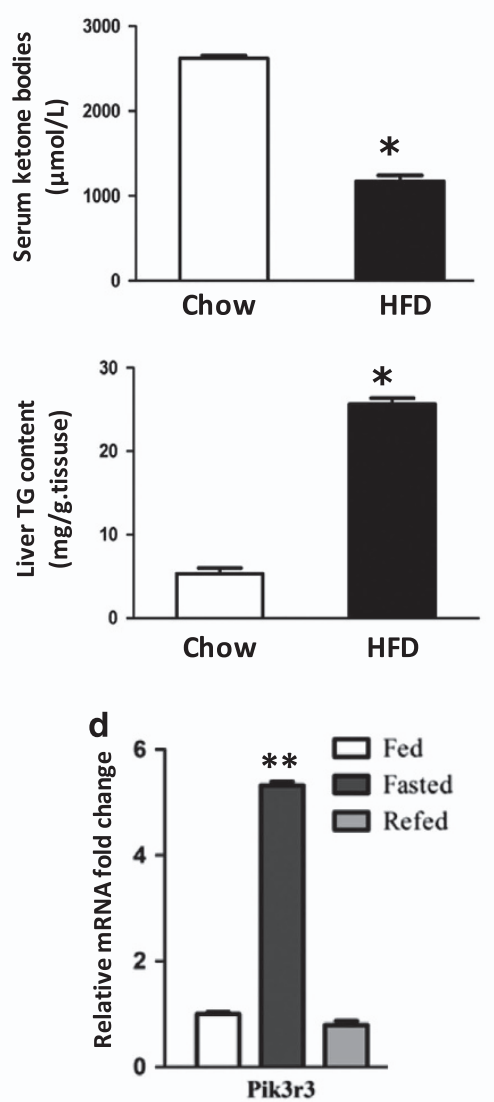

C
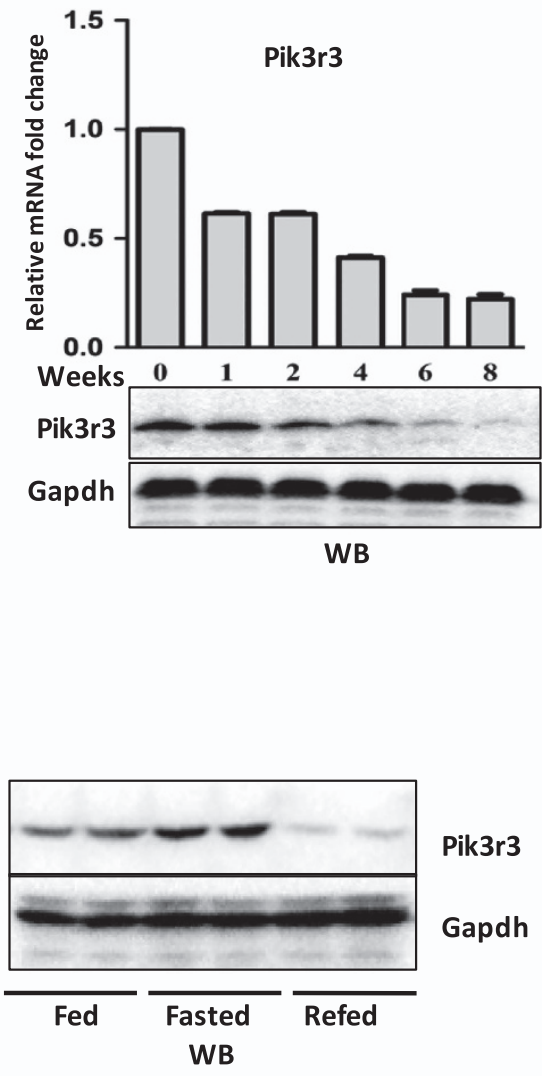

Pik3r3

Gapdh

Figure 1 PIK3R3 expression is regulated by nutritional status and is upregulated in HFD-induced fatty liver mice. (a) Representative morphology and H\&E, Oil Red O, Acadm and Cptla stained liver sections from C57BL/6J mice fed a normal chow or high-fat diet (HFD) for 8 weeks ( $n=6 /$ group). (b) Serum ketone body (top panel) and hepatic TG (bottom panel) levels of mice fed a normal chow diet or HFD. (c) Quantitative PCR (top panel) analysis and western blot (bottom panel) analysis showing the expression level changes of Pik3r3 in mice livers during HFD feeding. (d) Quantitative PCR (left) analysis and western blot (right) analysis showing expression levels of hepatic Pik3r3 in C57BL/6J mice subjected to ad libitum feeding, $24 \mathrm{~h}$ fasting and $24 \mathrm{~h}$ fasted/24 $\mathrm{h}$ re-fed conditions ( $n=2 / g r o u p)$. Data are expressed as the means \pm s.e.m. ${ }^{*} P<0.05 ;{ }^{*} P<0.01$

mRNA and protein expression levels in HFD-fed mice. The results showed that PIK3R3 was downregulated over time in mice fed the HFD at both the protein and the mRNA levels (Figure 1c, Supplementary Figure 1). We further investigated whether hepatic PIK3R3 expression could be regulated by nutritional status during the fasting/re-feeding cycle. Indeed, prolonged fasting $(24 \mathrm{~h})$ increased hepatic PIK3R3 mRNA and protein expression levels, whereas refeeding decreased these levels to those found at baseline (Figure 1d). Taken together, these data revealed a strong correlation between PIK3R3 expression and both lipid metabolism and nutritional status in the liver.

Overexpression of PIK3R3 regulated increases in fatty acid $\beta$-oxidation in hepatic cells and reduced fatty liver in vivo To identify the role of PIK3R3 in lipid metabolism, hepatic cells (HepG2 and LO2) were transfected with the PIK3R3 overexpression vector (PIK3R3) or the control vector (Vector). $\mathrm{CPT} 1 \mathrm{a}$ and ACADM protein and mRNA expression levels were upregulated in cells with PIK3R3 overexpression vs cells that were transfected with the control vector (Figure 2a; Supplementary Figure 2A). We next measured the levels of ketone bodies in the media of the transfected cells. Overexpression of PIK3R3 increased the level of ketone bodies (Supplementary Figure 2B), suggesting that PIK3R3 plays an important role in fatty acid oxidation. Real-time PCR results suggested that the overexpression of PIK3R3 increased the expression levels of genes involved in mitochondrial and peroxisomal fatty acid oxidation, such as Cptla, Acadm, Cyp4a10 and Cyp4a14 (Figure 2b). To investigate the role of PIK3R3 in hepatic lipid metabolism in vivo, we injected HFD mice with the Pik3r3 recombinant adenovirus vector (Ad-Pik3r3) or control adenovirus (Ad-control) via the tail vein to induce the overexpression of PIK3R3 in the liver. Remarkably, Ad-Pik3r3-infected HFD mice had a significantly improved fatty liver phenotype, as revealed by gross morphological changes and histological analyses (H\&E and Oil Red O staining) (Figure 2c). CPT1a and ACADM expression levels were also upregulated in Ad-Pik3r3-infected HFD mice (Figure 2c). Biochemical analyses also revealed a significant 
a
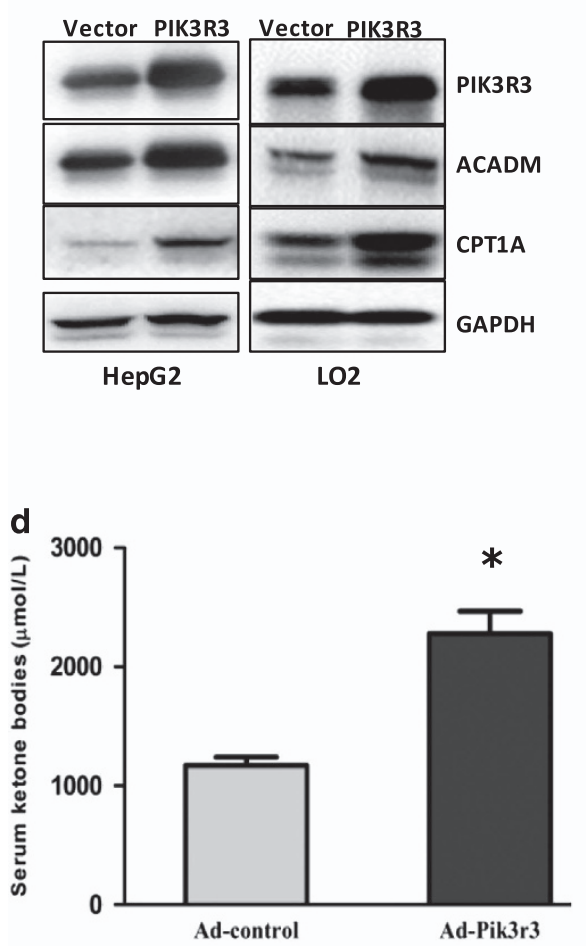

b

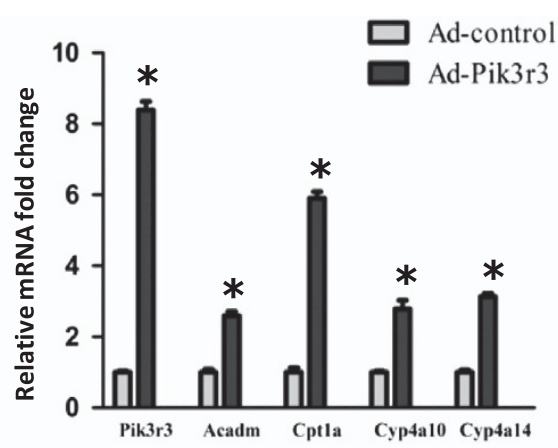

C

HE

Oil red

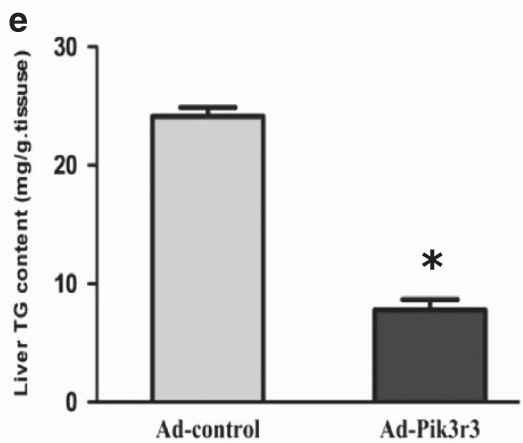

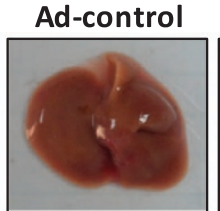

Ad-Pik3r3

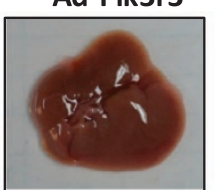

Acadm
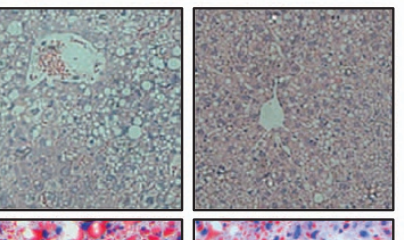

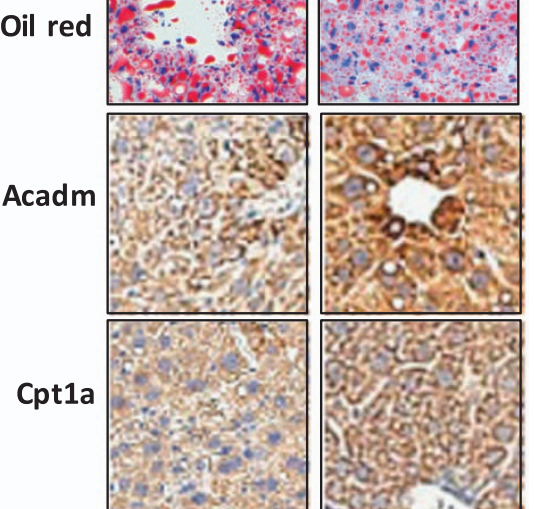

Figure 2 Overexpression of PIK3R3 regulated lipid metabolism in vitro and alleviated the fatty liver phenotype in vivo. (a) The indicated protein expression levels were detected by western blot after hepatic HepG2 and LO2 cells were transfected with the PIK3R3 overexpression vector (PIK3R3) or control vector (Vector). (b) Quantitative PCR analysis and chemical colorimetric diagnostic analysis showing the levels of genes involved in fatty acid oxidation in mice livers. (c) Mice were fed HFD for 8 weeks ( $n=6 / g r o u p)$ and injected with Ad-control or Ad-Pik3r3 as described in the Materials and methods. Mice were killed 5 days after the last injection. Representative morphology and H\&E, Oil Red O, Acadm and Cptla stained sections of mouse livers. (d, e) Serum ketone body levels (d) and hepatic TG levels (e). Data are expressed as the means \pm s.e.m. ${ }^{*} P<0.05$.

decrease in serum ketone bodies and hepatic TG levels in Ad-Pik3r3-infected HFD mice compared with control Ad-control-infected HFD mice (Figure $2 \mathrm{~d}$ and e).

\section{Knockdown of PIK3R3 impairs hepatic fatty acid} $\beta$-oxidation in cell culture and in vivo

We then transfected the hepatic cell lines HepG2 and LO2 with PIK3R3 siRNA (si-PIK3R3) to knock down PIK3R3 expression and determined the protein expression levels of PIK3R3, CPT1a and ACADM by immunoblotting. When PIK3R3 expression was knocked down, CPTla and ACADM protein levels were downregulated compared with those in the cells transfected with control siRNA (si-control) (Figure 3a). CPT1a and ACADM mRNA levels were also downregulated by si-PIK3R3 (Supplementary Figure 3A). The levels of ketone bodies in the cell media from cells treated with si-PIK3R3 or si-control showed that the downregulation of PIK3R3 decreased the levels of ketone bodies (Supplementary Figure 3B). These data further demonstrated that PIK3R3 plays an important role in fatty acid $\beta$-oxidation in hepatic cells. Real-time PCR results suggested that the downregulation of PIK3R3 also decreased the expression levels of genes involved in mitochondrial and peroxisomal fatty acid oxidation, such as Cpt1a, Acadm, Cyp4a10 and Cyp4a14 (Figure $3 \mathrm{~b}$ ). To examine the effects of the loss of PIK3R3 in vivo, mice were fed normal chow and infected with si-Pik3r3 by tail vein injection to inhibit Pik3r3 expression in the liver. Interestingly, these normal chow-fed mice infected with si-Pik3r3 developed a severe fatty liver phenotype (Figure 3c). Immunostaining showed that the Cptla and Acadm protein expression levels were also downregulated by si-Pik3r3 (Figure 3c). Biochemical analyses revealed a significant decrease in serum ketone bodies and an increase in hepatic TG levels in mice fed the normal chow diet and treated with si-Pik3r3 compared with those treated with si-control (Figure 3d and e).

\section{PIK3R3 regulates the expression of PPAR $\alpha$}

PPAR $\alpha$ and its target genes have been shown to play key roles in hepatic lipid metabolism. ${ }^{19}$ We thus examined the relationship between Pparo mRNA/protein expression levels in mice fed normal chow and mice fed a HFD. Interestingly, Ppar $\alpha$ mRNA and protein expression levels were downregulated in HFD-fed mice (Figure 4a), consistent with its important role in lipid metabolism. Moreover, adenovirus overexpression of PIK3R3 increased the expression of PPAR $\alpha$ in the livers of HFD-fed mice at both the mRNA and the protein 
a
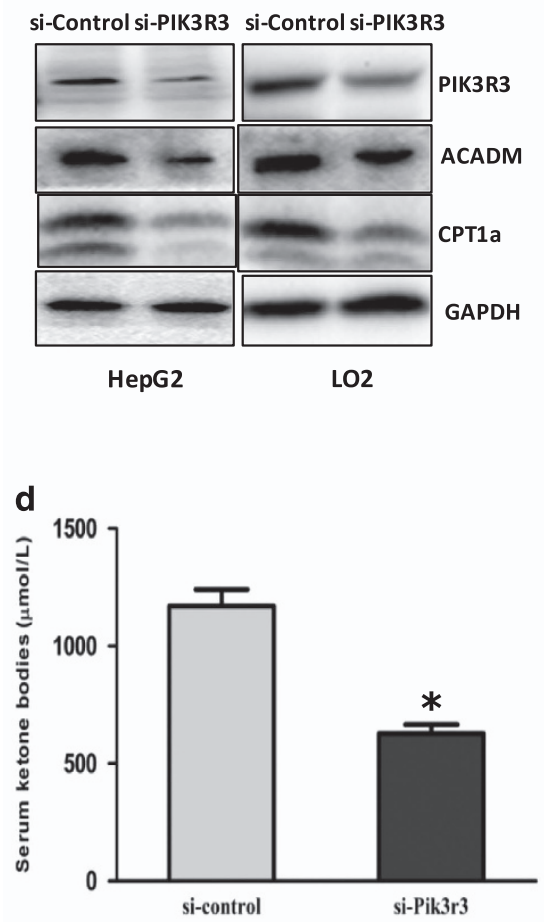

b
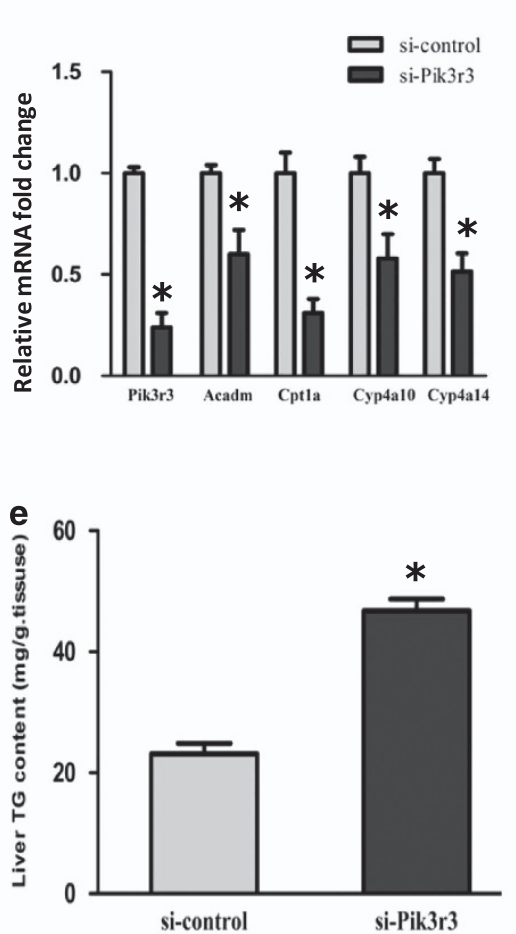

C

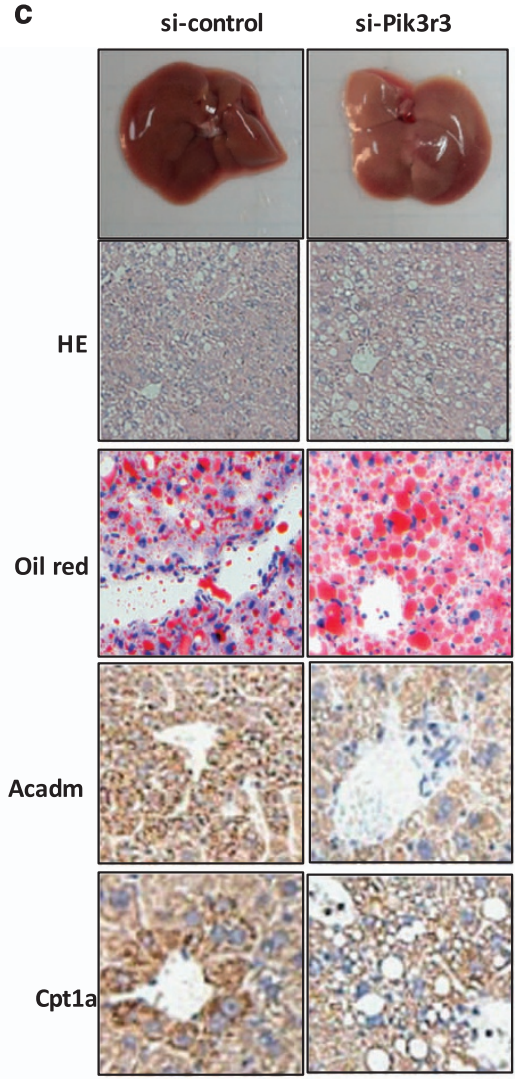

Figure 3 Down-regulation of PIK3R3 impairs lipid homeostasis in vitro and in vivo. (a) PIK3R3 was knocked down in hepatic HepG2 and LO2 cells by siRNA transfection; the indicated protein expression levels were detected by western blot. (b) Quantitative PCR analysis and chemical colorimetric diagnostic analysis showing the levels of genes involved in fatty acid oxidation in mice livers. (c) Mice were fed HFD for 8 weeks ( $n=6$ /group) and injected with si-control or si-Pik3r3 as described in the Materials and methods. Mice were killed 5 days after the last injection. Representative morphology and H\&E, Oil Red O, Acadm and Cptla stained sections of mouse livers. (d, e) Serum ketone body levels (d) and hepatic TG levels (e). Data are expressed as the means \pm s.e.m. ${ }^{*} P<0.05$.

levels (Figure $4 \mathrm{~b}$ and c, Supplementary Figure 4A), and downregulation of PIK3R3 decreased the expression of PPAR $\alpha$ in normal chow-fed mice at both the protein and the mRNA levels (Figure 4d and e, Supplementary Figure 4B). Furthermore, siRNA-mediated knockdown of PIK3R3 in LO2 and HepG2 cells decreased the expression of PPAR $\alpha$ but had no effect on PPAR $\gamma$ (Figure 4f, Supplementary Figure 4C). Adenovirus-mediated overexpression of PIK3R3 induced the expression of PPAR $\alpha$ but had no effect on the expression of PPAR $\gamma$ (Figure 4g, Supplementary Figure 4D). Taken together, our data showed that PIK3R3 controls the expression of PPAR $\alpha$. Further supporting the role of PIK3R3 in the nutritional regulation of PPAR $\alpha$ expression, mice were subjected to a fasting/feeding cycle. Hepatic Ppar $\alpha$ mRNA and protein expression levels increased after prolonged fasting $(24 \mathrm{~h})$. These changes were reversed by refeeding (Supplementary Figure 4E).

\section{PPAR $\alpha$ mediates the effects of PIK3R3 on cellular and hepatic lipid metabolism}

To test whether PPAR $\alpha$ mediates the PIK3R3 effects on hepatic lipid metabolism, we performed PPAR $\alpha$ rescue and inhibition experiments in hepatic cells. The simultaneous overexpression of PIK3R3 and knockdown of PPAR $\alpha$ expression by si-PPAR $\alpha$ decreased the expression of PPAR $\alpha$ and its target genes ACADM and CPT1a in LO2 cells (Figure 5a). The levels of ketone bodies in the cell supernatants were also reversed by co-treatment with PIK3R3 and si-PPAR $\alpha$ in LO2 cells (Supplementary Figure 5A). The knockdown of PIK3R3 in HepG2 cells also decreased the expression of PPAR $\alpha$ and its target genes ACADM and CPT1a. Transfection of the PPAR $\alpha$ vector in the PIK3R3 knockdown cells restored the expression of ACADM and CPT1a (Figure 5b), as well as the levels of ketone bodies in the supernatants (Supplementary Figure 5B). To further test whether PPAR $\alpha$ mediates the effects of PIK3R3 on hepatic lipid metabolism in vivo, si-Pparo was injected via the tail vein into Ad-Pik3r3-infected HFD mice. si-Pparo significantly inhibited the Pik3r3 induction of Pparo and its target genes involved in hepatic fatty acid oxidation (Figure $5 \mathrm{c}$ and d, Supplementary Figure 5C) and almost completely abolished the ability of PIK3R3 to reduce hepatic TG content and to increase serum ketone bodies (Figure $5 \mathrm{e}$ and $\mathrm{f}$ ). In addition, we also measured the body weights of the mice in each group; the data showed that the overexpression of Pik3r3 could reduce the body weights of HFD-fed mice, compared with the control group, and this body weight loss could be rescued by the knockdown of Ppara (Supplementary 


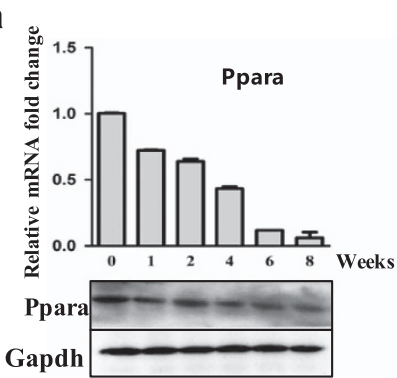

b

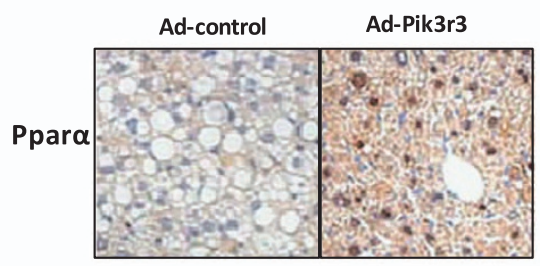

c

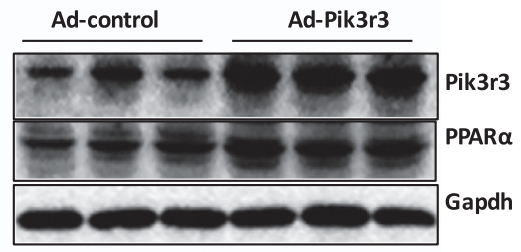

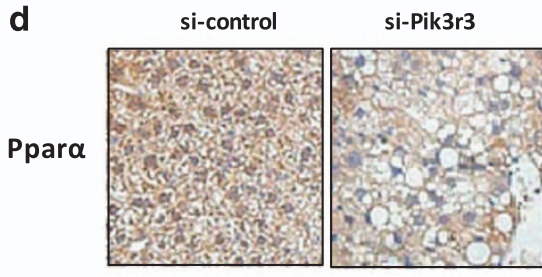

f

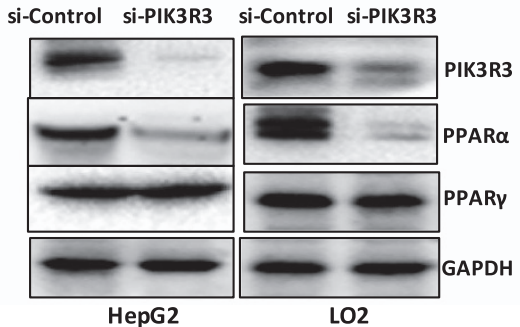

e

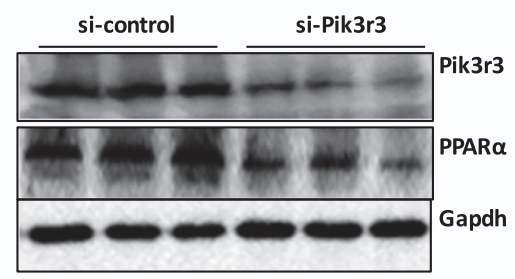

g

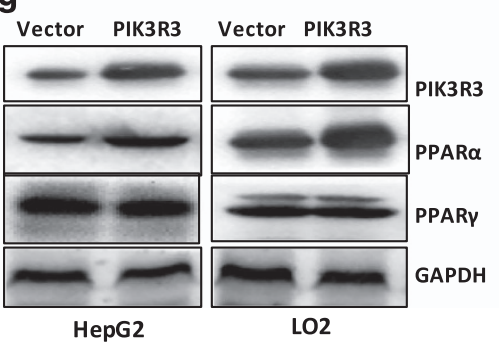

Figure 4 PIK3R3 regulates the expression of PPAR $\alpha$. (a) Quantitative PCR (upper panel) analysis and western blot (bottom panel) analysis showing the expression level changes of Ppara in mice livers during HFD feeding. Data are expressed as the means \pm s.e.m. ${ }^{*} P<0.05$; ${ }^{*} P<0.01$. (b) Immunohistochemistry analysis showing the expression level changes of hepatic Pparo in the livers of the mice in Figure 2c. (c) Western blot analysis showing the expression level changes of hepatic Pparo in the livers of the mice in Figure 2c. (d) Immunohistochemistry analysis showing the expression level changes of hepatic Ppara in the livers of the mice in Figure 3c. (e) Western blot analysis showing the expression level changes of hepatic Pparo in the livers of the mice in Figure 3c. (f) PIK3R3 was knocked down in HepG2 and L02 cells by siRNAs transfection. Western blot analysis showing the expression levels of PPARA and PPARG. (g) PIK3R3 was overexpressed in hepatic HepG2 and LO2 cells by plasmid transfection. Western blot analysis showing the expression levels of PPARA and PPARG.

Figure 5D). Conversely, the overexpression of Ppar $\alpha$ in si-Pik3r3-treated mice receiving the normal chow diet and transfected with Ad-Pparo significantly reversed the inhibitory effects of si-Pik3r3 on Pparo expression and its fatty acid oxidation genes in the liver (Supplementary Figure 6A-C). The overexpression of Ppara also reversed the effects of si-Pik3r3 on hepatic TG content and serum ketone bodies (Supplementary Figure 6D and E). Taken together, these data show that PPAR $\alpha$ signaling plays a critical role in mediating the effects of PIK3R3 on hepatic lipid homeostasis.

PIK3R3 regulation of PPAR $\alpha$ expression depends on HNF4 $\alpha$ Our data showed that PIK3R3 induced both PPAR $\alpha$ mRNA and protein levels, suggesting that PIK3R3 may regulate PPAR $\alpha$ gene expression. HNF4 $\alpha$ and NR2F2 were previously reported to be the main transcription factors that bound to the PPAR $\alpha$ promoter and regulated the gene expression of $\mathrm{PPaR}^{22}$ Accordingly, we examined the effects of PIK3R3 on HNF4 $\alpha$ and NR2F2 expression in hepatic cells and found that HNF4 $\alpha$ expression was upregulated in cells overexpressing PIK3R3 and downregulated in PIK3R3-deficient cells (Figure 6a, Supplementary Figure 7A). There was little change in NR2F2 expression under these conditions. To demonstrate the critical role of HNF4a in the PIK3R3 regulation of PPAR $\alpha$ gene expression, we co-transfected LO2 cells with si-HNF4 $\alpha$ and PIK3R3 vectors and found that knockdown of HNF4 $\alpha$ expression abrogated the induction of PPAR $\alpha$ expression due to PIK3R3 overexpression (Figure 6b, Supplementary Figure 7B). Furthermore, in LO2 cells co-transfected with the HNF4 $\alpha$ vector and si-PIK3R3, the decreased PPAR $\alpha$ expression due to PIK3R3 knockdown was rescued (Figure 6c, Supplementary Figure 7C). To further investigate the role of HNF4 $\alpha$ in PIK3R3-regulated PPAR $\alpha$ expression, we examined HNF4 $\alpha$ binding to the PPAR $\alpha$ promoter using chromatin immunoprecipitation (ChIP) assays. Interestingly, the overexpression of PIK3R3 promoted HNF4 $\alpha$ binding to the PPAR $\alpha$ promoter, whereas PIK3R3 knockdown inhibited the binding of HNF4 $\alpha$ to the PPAR $\alpha$ promoter (Figure $6 \mathrm{~d}$ and e). These data suggest that PIK3R3 regulation of PPAR $\alpha$ expression depends upon its induction of HNF4 $\alpha$. 
a

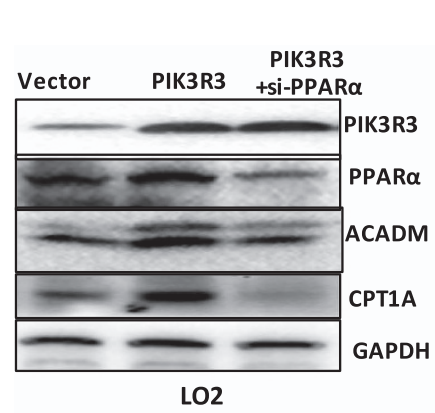

b

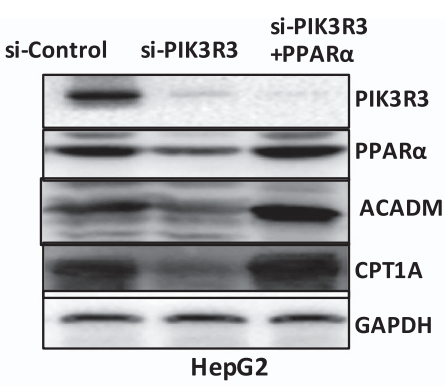

d

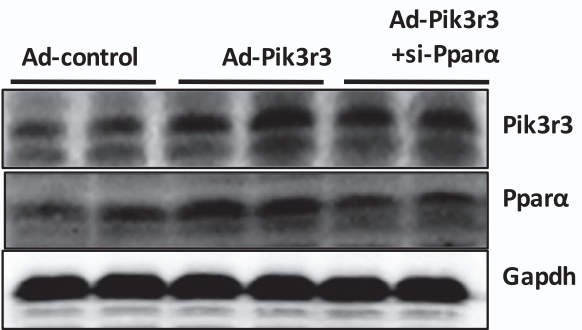

Ad-Pik3r3
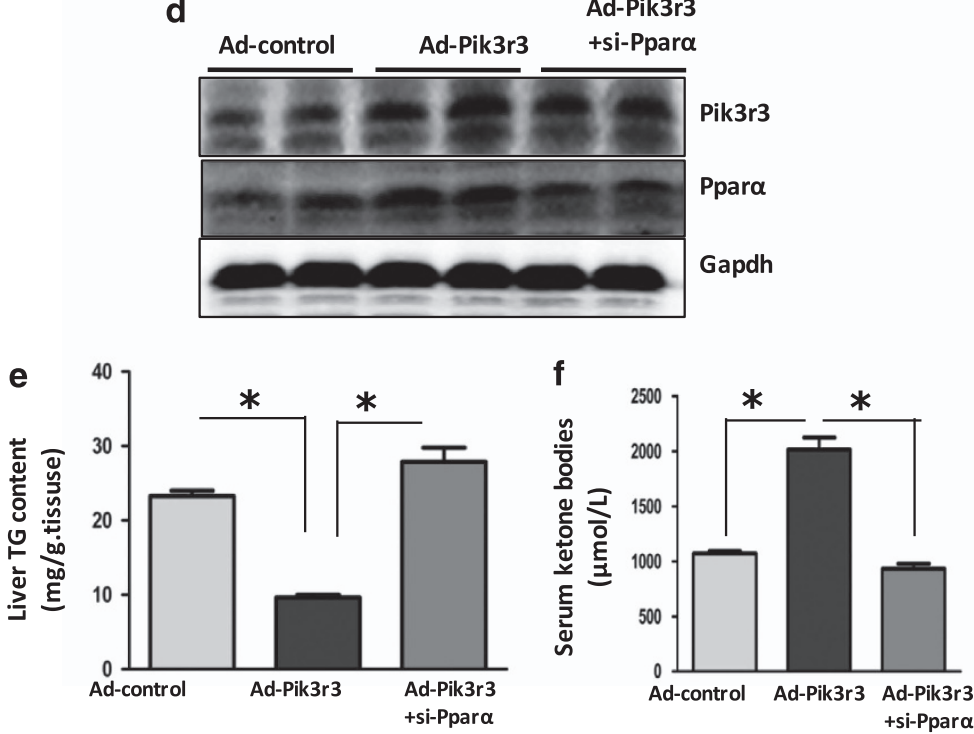

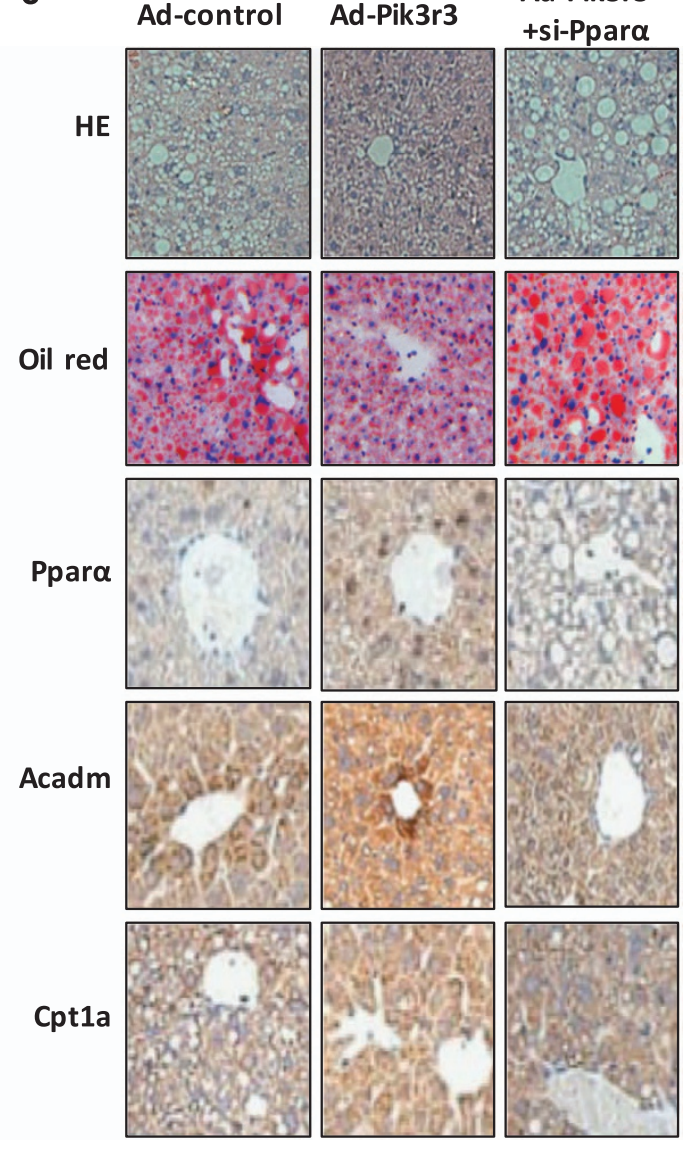

Figure 5 PPAR $\alpha$ mediates the effects of PIK3R3 on cellular and hepatic lipid metabolism. (a) PIK3R3 was overexpressed in LO2 cells with or without the knockdown of PPARA; western blot analysis showing the protein levels of PIK3R3, PPARA, ACADM and CPT1A. (b) PIK3R3 was knocked down in HepG2 cells with or without the overexpression of PPARA; western blot analysis showing the protein levels of PIK3R3, PPARA, ACADM and CPT1A. (c) Mice were fed HFD for 8 weeks ( $n=6 /$ group) and injected with Ad-control, Ad-Pik3r3 or Ad-Pik3r3+si-Ppara as described in the Materials and methods. Mice were killed for further analysis 5 days after the last injection. Representative H\&E, Oil Red O, Ppara, Acadm and Cptla stained sections of mouse livers. (d) Pik3r3 and Ppar $\alpha$ expression levels were detected by western blot. (e) Chemical colorimetric diagnostic analysis showing the changes in hepatic TG. (f) Chemical colorimetric diagnostic analysis showing changes in serum ketone bodies. Data are expressed as the means \pm s.e.m. ${ }^{*} P<0.05$.

\section{DISCUSSION}

The phosphatidylinositol 3-kinase (PI3K)/Akt signaling pathway plays an important role in a variety of biological processes, such as cell metabolism, glucose transporter regulation, cell cycle regulation, cell growth, and cancer development. ${ }^{4,23}$ The activation of Akt suppresses the expression of genes involved in hepatic fatty acid $\beta$-oxidation and decreases hepatic gluconeogenesis. $^{24}$ It was previously reported that sterolregulatory element binding proteins (SREBPs), key players in fatty acid and cholesterol biosynthesis, regulate the expression of PIK3R3, a PI3K regulatory subunit, to help mediate metabolic responses to insulin and growth factors in various tissues. ${ }^{25}$ Previously, little was known about the downstream signaling pathway and role of PIK3R3 in hepatic lipid metabolism. In this study, we described a novel role and mechanism for PIK3R3 in hepatic lipid metabolism. In vivo and in vitro evidence suggested that PIK3R3 surprisingly promoted hepatic fatty acid $\beta$-oxidation rather than lipogenesis via the induction of PPAR $\alpha$ expression and its target genes. In particular, tail vein injection of an adenoviral vector expressing Pik3r3 markedly reduced fatty liver and improved hepatic fatty acid $\beta$-oxidation in mice fed a HFD. By contrast, the transfection of Pik3r3 siRNA induced fatty liver and decreased hepatic fatty acid $\beta$-oxidation in mice fed a normal chow diet. Moreover, PIK3R3 mRNA and protein expression levels were decreased in the fatty livers from mice fed the HFD, suggesting that reduced PIK3R3 may contribute to the development of hepatosteatosis. These findings suggest that PIK3R3 expression may play an important compensatory role in opposing the lipogenic actions of insulin through its stimulation of fatty acid $\beta$-oxidation.

$\operatorname{PPAR} \alpha, \operatorname{PPAR} \gamma$ and $\operatorname{PPAR} \beta / \delta$ are ligand-activated transcription factors belonging to the NR1C nuclear receptor subfamily. ${ }^{26,27}$ Many PPAR target genes are involved in fatty acid metabolism in tissues with high rates of oxygen consumption, such as the muscle, heart and liver. ${ }^{19}$ PPAR $\alpha$ activation 
a

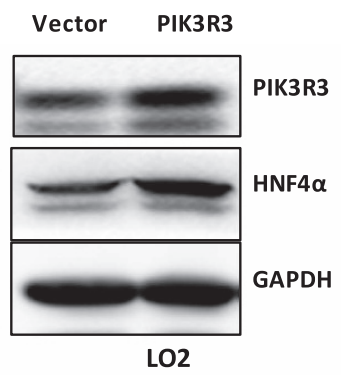

b

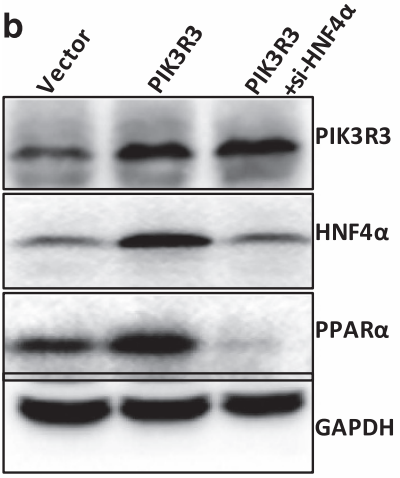

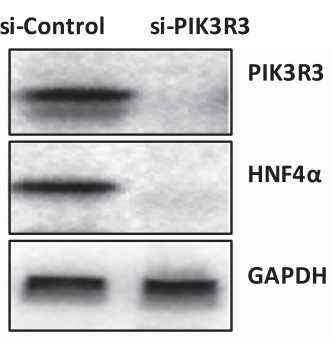

HepG2

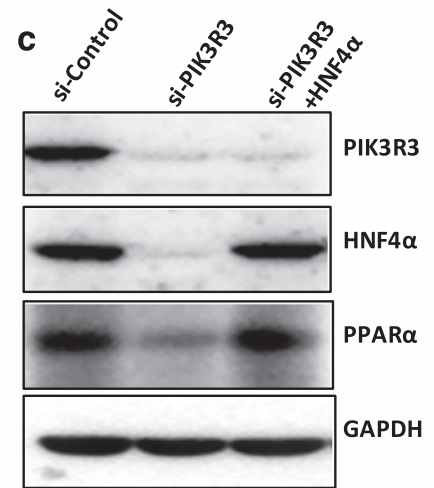

d

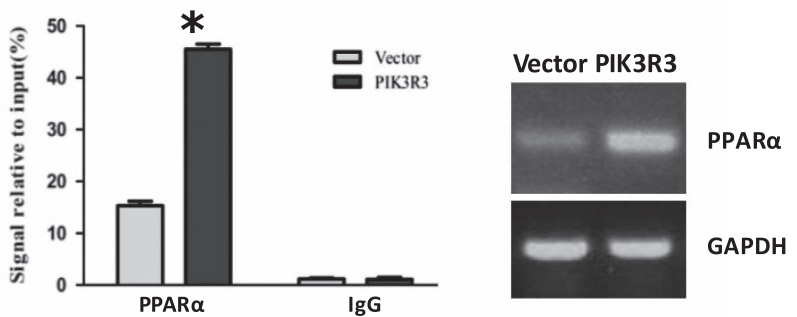

e

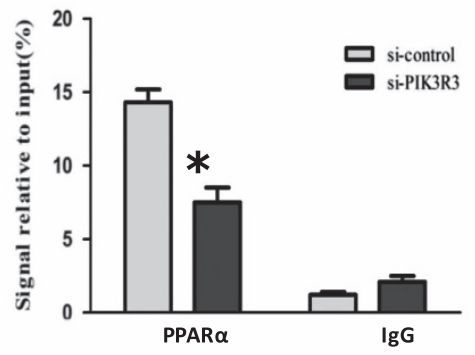

Figure 6 PIK3R3 regulation of PPAR $\alpha$ expression was dependent on HNF4 $\alpha$. (a) PIK3R3 was overexpressed in LO2 cells and knocked down in HepG2 cells; western blots were used to detect the protein levels of PIK3R3 and HNF4 $\alpha$. (b) PIK3R3 was overexpressed in LO2 cells with or without HNF4 $\alpha$ knockdown; western blot analysis showing the expression levels of PIK3R3, HNF4 $\alpha$ and PPAR $\alpha$. (c) PIK3R3 was knocked down in LO2 cells with or without HNF4 $\alpha$ overexpression; western blot analysis showing the expression levels of PIK3R3, HNF4 $\alpha$ and PPAR $\alpha$. (d) ChIP assay and quantitative PCR analysis showing the binding activity of HNF4 $\alpha$ to the PPAR $\alpha$ promoter after the overexpression of PIK3R3. (e) ChIP assay and quantitative PCR analysis showing the binding activity of HNF4 $\alpha$ to the PPAR $\alpha$ promoter after the downregulation of PIK3R3. Data are expressed as the means \pm s.e.m. ${ }^{*} P<0.05$.

improves steatosis, inflammation and fibrosis in pre-clinical models of non-alcoholic fatty liver disease and has been identified as a new potential therapeutic area. ${ }^{2}$ Some PPAR $\alpha$ agonists have been studied as potential candidate drugs for non-alcoholic fatty liver disease therapy in humans. ${ }^{28,29}$ Our study showed that PIK3R3 induced HNF4 $\alpha$ expression, a transcription factor involved in the regulation of serum glucose and lipid levels. HNF4 $\alpha$ is also a key transcription factor mediating PPAR $\alpha$ expression; however, the precise mechanism by which PIK3R3 stimulates the gene expression of HNF4a is still not known. PPAR $\alpha$ and HNF4 $\alpha$ rescue and inhibition experiments in hepatic cells and in vivo provided further evidence for a PIK3R3-HNF4 $\alpha$-PPAR $\alpha$ pathway that plays a significant role in hepatic lipid metabolism. A better understanding of this signaling pathway, particularly the regulation of PIK3R3 expression in different nutritional states, may help novel molecular targets for treating non-alcoholic fatty liver disease to be determined.

Currently, PI3K inhibitors are mainly used for anti-cancer therapy; however, only a subset of the many cellular processes regulated by the PI3K pathway is directly involved in cell proliferation. ${ }^{30}$ Recently, the pharmacological inhibition of PI3K has been shown to be an effective and safe anti-obesity intervention that could reverse the negative effects of metabolic syndrome in humans. ${ }^{5}$ Previously, we reported that PIK3R3 contained a unique $\mathrm{NH}_{2}$ terminus that mediated
PIK3R3-specific functions that were different from other regulatory subunits. ${ }^{31}$ Some of the known functions of PIK3R3 are as follows. Our findings with Rb and PCNA, as well as earlier studies of PIK3R3 (Morris White), may help us to determine potential reasons why nutritional status might regulate PIK3R3. Our previous studies have suggested that targeting PIK3R3 regulatory subunits could potentially facilitate the development of a new class of drugs that may have potential advantages over present PI3K catalytic subunit inhibitors. ${ }^{12,31}$ In summary, we have identified and characterized an important role for PIK3R3 in hepatic lipid metabolism. We have also identified a novel PIK3R3-HNF4 $\alpha-P P A R \alpha$ signaling pathway that appears to be a key contributor to hepatic fatty acid $\beta$-oxidation. As the induction of PIK3R3 improved the fatty liver phenotype by increasing fatty acid $\beta$-oxidation, targeting PIK3R3 may be an effective strategy for treating NAFLD, a condition for which there is currently no approved drug therapy.

\section{CONFLICT OF INTEREST}

The authors declare no conflict of interest.

\section{ACKNOWLEDGEMENTS}

Supported by the National Natural Science Foundation (no. 81570525) and the Program for Changjiang Scholars and 
Innovative Research Team at Huazhong University of Science and Technology (IRT_14R20).

Author contributions: XY, YF, FH and XL performed the experiments; $\mathrm{JH}, \mathrm{GW}$ and XY analyzed the results and made the figures; GW, XY and $\mathrm{JH}$ wrote the paper; $\mathrm{GW}$ and $\mathrm{JH}$ designed the research.

\section{PUBLISHER'S NOTE}

Springer Nature remains neutral with regard to jurisdictional claims in published maps and institutional affiliations.

1 Browning JD, Horton JD. Molecular mediators of hepatic steatosis and liver injury. J Clin Invest 2004; 114: 147-152.

2 Pawlak M, Lefebvre P, Staels B. Molecular mechanism of PPARalpha action and its impact on lipid metabolism, inflammation and fibrosis in nonalcoholic fatty liver disease. J Hepatol 2015; 62: 720-733.

3 Foukas LC, Berenjeno IM, Gray A, Khwaja A, Vanhaesebroeck B. Activity of any class IA PI3K isoform can sustain cell proliferation and survival. Proc Natl Acad Sci USA 2010; 107: 11381-11386.

4 Vanhaesebroeck B, Guillermet-Guibert J, Graupera M, Bilanges B. The emerging mechanisms of isoform-specific PI3K signalling. Nat Rev Mol Cell Biol 2010; 11: 329-341.

5 Ortega-Molina A, Lopez-Guadamillas E, Mattison JA, Mitchell SJ, Munoz-Martin M, Iglesias $G$ et al. Pharmacological inhibition of PI3K reduces adiposity and metabolic syndrome in obese mice and rhesus monkeys. Cell Metab 2015; 21: 558-570.

6 Krycer JR, Sharpe LJ, Luu W, Brown AJ. The Akt-SREBP nexus: cell signaling meets lipid metabolism. Trends Endocrinol Metab 2010; 21: 268-276.

7 Yamauchi Y, Furukawa K, Hamamura K, Furukawa K. Positive feedback loop between PI3K-Akt-mTORC1 signaling and the lipogenic pathway boosts Akt signaling: induction of the lipogenic pathway by a melanoma antigen. Cancer Res 2011; 71: 4989-4997.

8 Sopasakis VR, Liu P, Suzuki R, Kondo T, Winnay J, Tran TT et al. Specific roles of the p110alpha isoform of phosphatidylinsositol 3-kinase in hepatic insulin signaling and metabolic regulation. Cell Metab 2010; 11: 220-230.

9 Pons S, Asano T, Glasheen E, Miralpeix M, Zhang Y, Fisher TL et al. The structure and function of p55PIK reveal a new regulatory subunit for phosphatidylinositol 3-kinase. Mol Cell Biol 1995; 15: 4453-4465.

10 Wang G, Chen C, Yang R, Cao X, Lai S, Luo X et al. p55PIK-PI3K stimulates angiogenesis in colorectal cancer cell by activating NF-kappaB pathway. Angiogenesis 2013; 16: 561-573.

11 Wang G, Deng Y, Cao X, Lai S, Tong Y, Luo X et al. Blocking p55PIK signaling inhibits proliferation and induces differentiation of leukemia cells. Cell Death Differ 2012; 19: 1870-1879.

12 Wang G, Cao X, Lai S, Luo X, Feng Y, Xia X et al. PI3K stimulates DNA synthesis and cell-cycle progression via its p55PIK regulatory subunit interaction with PCNA. Mol Cancer Ther 2013; 12: 2100-2109.

13 Xia X, Cheng A, Akinmade D, Hamburger AW. The N-terminal 24 amino acids of the p55 gamma regulatory subunit of phosphoinositide 3-kinase binds $\mathrm{Rb}$ and induces cell cycle arrest. Mol Cell Biol 2003; 23: 1717-1725.

14 Wang G, Cao X, Lai S, Luo X, Feng Y, Wu J et al. Altered p53 regulation of miR-148b and p55PIK contributes to tumor progression in colorectal cancer. Oncogene 2015; 34: 912-921.

15 Kersten S, Seydoux J, Peters JM, Gonzalez FJ, Desvergne B, Wahli W. Peroxisome proliferator-activated receptor alpha mediates the adaptive response to fasting. J Clin Invest 1999; 103: 1489-1498.

16 Pawlak M, Bauge E, Bourguet W, De Bosscher K, Lalloyer F, Tailleux A et al. The transrepressive activity of peroxisome proliferator-activated receptor alpha is necessary and sufficient to prevent liver fibrosis in mice. Hepatology 2014; 60: 1593-1606.

17 Leone TC, Weinheimer CJ, Kelly DP. A critical role for the peroxisome proliferator-activated receptor alpha (PPARalpha) in the cellular fasting response: the PPARalpha-null mouse as a model of fatty acid oxidation disorders. Proc Natl Acad Sci USA 1999; 96: 7473-7478.

18 Ip E, Farrell GC, Robertson G, Hall P, Kirsch R, Leclercq I. Central role of PPARalpha-dependent hepatic lipid turnover in dietary steatohepatitis in mice. Hepatology 2003; 38: 123-132.

19 Rakhshandehroo M, Knoch B, Muller M, Kersten S. Peroxisome proliferatoractivated receptor alpha target genes. PPAR Res 2010; 2010: 612089.

20 Kersten S, Mandard S, Escher P, Gonzalez FJ, Tafuri S, Desvergne B et al. The peroxisome proliferator-activated receptor alpha regulates amino acid metabolism. FASEB J 2001; 15: 1971-1978.

$21 \mathrm{Li} \mathrm{T}$, Chiang JY. Regulation of bile acid and cholesterol metabolism by PPARs. PPAR Res 2009; 2009: 501739.

22 McMullen PD, Bhattacharya S, Woods CG, Sun B, Yarborough K, Ross SM et al. A map of the PPARalpha transcription regulatory network for primary human hepatocytes. Chem Biol Interact 2014; 209: 14-24.

23 Marone R, Cmiljanovic V, Giese B, Wymann MP. Targeting phosphoinositide 3-kinase: moving towards therapy. Biochim Biophys Acta 2008; 1784: $159-185$.

24 Li X, Monks B, Ge Q, Birnbaum MJ. Akt/PKB regulates hepatic metabolism by directly inhibiting PGC-1alpha transcription coactivator. Nature 2007; 447: 1012-1016.

25 Kallin A, Johannessen LE, Cani PD, Marbehant CY, Essaghir A, Foufelle F et al. SREBP-1 regulates the expression of heme oxygenase 1 and the phosphatidylinositol-3 kinase regulatory subunit p55 gamma. J Lipid Res 2007; 48: 1628-1636.

26 Issemann I, Green S. Activation of a member of the steroid hormone receptor superfamily by peroxisome proliferators. Nature 1990; 347: 645-650.

27 Kliewer SA, Forman BM, Blumberg B, Ong ES, Borgmeyer U, Mangelsdorf DJ et al. Differential expression and activation of a family of murine peroxisome proliferator-activated receptors. Proc Natl Acad Sci USA 1994; 91: 7355-7359.

28 Staels B, Maes M, Zambon A. Fibrates and future PPARalpha agonists in the treatment of cardiovascular disease. Nat Clin Pract Cardiovasc Med 2008; 5: 542-553.

29 Staels B, Rubenstrunk A, Noel B, Rigou G, Delataille P, Millatt LJ et al. Hepatoprotective effects of the dual peroxisome proliferator-activated receptor alpha/delta agonist, GFT505, in rodent models of nonalcoholic fatty liver disease/nonalcoholic steatohepatitis. Hepatology 2013; 58: 1941-1952.

30 Fruman DA, Rommel C. PI3K and cancer: lessons, challenges and opportunities. Nat Rev Drug Discov 2014; 13: 140-156.

$31 \mathrm{Hu}$ J, Xia X, Cheng A, Wang G, Luo X, Reed MF et al. A peptide inhibitor derived from p55PIK phosphatidylinositol 3-kinase regulatory subunit: a novel cancer therapy. Mol Cancer Ther 2008; 7: 3719-3728.

This work is licensed under a Creative Commons Attribution-NonCommercial-NoDerivs 4.0 International License. The images or other third party material in this article are included in the article's Creative Commons license, unless indicated otherwise in the credit line; if the material is not included under the Creative Commons license, users will need to obtain permission from the license holder to reproduce the material. To view a copy of this license, visit http://creativecommons.org/licenses/by-nc-nd/4.0/

(C) The Author(s) 2018

Supplementary Information accompanies the paper on Experimental \& Molecular Medicine website (http://www.nature.com/emm) 